\title{
Polyethylenimine-functionalized silver nanoparticle-based co-delivery of paclitaxel to induce HepG2 cell apoptosis
}

This article was published in the following Dove Press journal:

International Journal of Nanomedicine

8 December 2016

Number of times this article has been viewed

\author{
Yinghua $\mathrm{Li}^{1, *}$ \\ Min Guo',* \\ Zhengfang Lin' \\ Mingqi Zhao' \\ Misi Xiao' \\ Changbing Wang' \\ Tiantian $\mathrm{Xu}^{\prime}$ \\ Tianfeng Chen ${ }^{2}$ \\ Bing Zhu' \\ 'Center Laboratory, Guangzhou \\ Women and Children's Medical \\ Center, Guangzhou Medical University, \\ 2Department of Chemistry, Jinan \\ University, Guangzhou, People's \\ Republic of China \\ *These authors contributed equally \\ to this work
}

\begin{abstract}
Hepatocarcinoma is the third leading cause of cancer-related deaths around the world. Recently, a novel emerging nanosystem as anticancer therapeutic agents with intrinsic therapeutic properties has been widely used in various medical applications. In this study, surface decoration of functionalized silver nanoparticles (AgNPs) by polyethylenimine (PEI) and paclitaxel (PTX) was synthesized. The purpose of this study was to evaluate the effect of Ag@ PEI@PTX on cytotoxic and anticancer mechanism on HepG2 cells. The transmission electron microscope image and 3-(4,5-dimethylthiazol-2-yl)-2,5-diphenyltetrazolium bromide assay showed that Ag@PEI@PTX had satisfactory size distribution and high stability and selectivity between cancer and normal cells.Ag@PEI@PTX-induced HepG2 cell apoptosis was confirmed by accumulation of the sub-G1 cells population, translocation of phosphatidylserine, depletion of mitochondrial membrane potential, DNA fragmentation, caspase-3 activation, and poly(ADPribose) polymerase cleavage. Furthermore, Ag@PEI@PTX enhanced cytotoxic effects on HepG2 cells and triggered intracellular reactive oxygen species; the signaling pathways of AKT, p53, and MAPK were activated to advance cell apoptosis. In conclusion, the results reveal that $\mathrm{Ag} @$ PEI@PTX may provide useful information on Ag@PEI@PTX-induced HepG2 cell apoptosis and as appropriate candidate for chemotherapy of cancer.
\end{abstract}

Keywords: silver nanoparticles, polyethylenimine, paclitaxel, reactive oxygen species, apoptosis

\section{Introduction}

Hepatocellular carcinoma (HCC) is considered as one of the most common primary liver cancers around the world and the second leading cause of cancer-associated mortality with 75,000 cases increasing annually. ${ }^{1-3}$ Unfortunately, the diagnosis of early-stage HCC is difficult and the long-term prognosis remains unsatisfactory without efficient early detection and surveillance strategies. ${ }^{4,5}$ Meanwhile, as most cases with $\mathrm{HCC}$ are metastatic or advanced at the time of diagnosis, the local therapies including liver transplantation and surgical excision are unsuitable. ${ }^{6,7}$ Furthermore, chemotherapeutic is generally ineffective against advanced HCC, which has high metastatic potential. ${ }^{8}$ Finally, most conventional cancer therapeutics have a relatively short half-life and poor permeability, which may influence curative effect. ${ }^{9}$ Therefore, it is urgent to develop a novel systemic chemotherapy for HCC in medicine.

Recently, nanobiotechnology plays an important role in cancer therapy. ${ }^{10,11}$ It improves the efficiency of drug delivery, reduces side effects of drug, and strengthens molecular targeting in biomedical applications. ${ }^{12-14}$ Nanomaterial is considered to be a promising solution to overcome the above problems with the peculiar properties, 
including high stability; smart, controllable morphology; thermal properties; soluble behaviors in aqueous solution; and surface functionalization. ${ }^{15}$ Among them, metal nanoparticles, especially silver nanoparticles (AgNPs), are considered as one of the nanomaterials with the highest degree of commercialization. ${ }^{16}$ Furthermore, AgNPs have gained wide attention due to its potent activity, including antiviral, antibacterial, antifungal, antiangiogenic, and antiinflammatory. ${ }^{17,18}$ In addition, several studies reported that AgNPs induced cell apoptosis in cancer cells but not in normal cells, as the cancer cells are more acidic, which is useful for the release of silver ions from the AgNPs. ${ }^{18,19}$ As one of the most traditional polycationic polymers, polyethylenimine (PEI), can promote nanoparticles entering into the cells by improving its zeta potential. ${ }^{20}$ PEI-functionalized AgNPs were chosen to be as drug carrier in our study. Paclitaxel (PTX), a chemotherapeutic agent, is widely used against several types of solid tumors besides breast, ovarian, lung, colon, head, neck, and liver cancers. ${ }^{21-27}$ PTX disrupts the tubulin-microtubule equilibrium, which is different from conventional anticancer drugs affecting nucleic acid synthesis to induce cancer cell death. ${ }^{28,29}$ It is considered as an appropriate candidate for chemotherapy because PTX at low concentrations can exert antiangiogenic activity. ${ }^{30}$ However, the major problem for the clinical efficacy of PTX is compromised by its poor water solubility, low permeability, and serious adverse effects. ${ }^{31}$ Therefore, to improve the anticancer efficacy,Ag@PEI@PTX was synthesized by PEI-conjugated AgNPs to induce cancer cell apoptosis.

Reactive oxygen species (ROS) were considered as one of the key players in some physiological processes through the mechanisms of apoptosis and autophagy. ${ }^{32-37}$ ROS are generated in several cellular systems, such as plasma membrane, peroxisomes, cytosol, endoplasmic reticulum, and membranes of mitochondria. ${ }^{37}$ The imbalance between ROS generation and antioxidant system triggers oxidative stress, which is related to much pathology, including cancer, diabetes, rheumatoid arthritis, cardiovascular disease, and other diseases. ${ }^{38,39}$ Many reports have demonstrated the relationship of AgNPsmediated cytotoxic and oxidative stress, but the anticancer mechanisms of AgNPs have remained poorly understood. ${ }^{40,41}$ Thus, Ag@PEI@PTX was synthesized as a novel chemotherapeutic agent to achieve HepG2 apoptosis.

\section{Materials and methods Materials}

The HepG2 cells were obtained from American Type

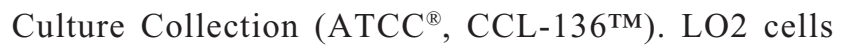
(normal human liver cells line) were provided from Cell
Bank of the Chinese Academy of Sciences (Shanghai, People's Republic of China). Dulbecco's Modified Eagle's Medium and fetal bovine serum purchased from Gibco were used for cell culture. PTX, $\mathrm{AgNO}_{3}$, vitamin C, branched PEI, propidium iodide (PI), 2', $7^{\prime}$-dichlorofluorescein diacetate, 4'6-diamidino-2-phenylindole (DAPI), 3-(4,5dimethylthiazol-2-yl)-2,5-diphenyltetrazolium bromide (MTT) were all obtained from Sigma. Capase-3, poly(ADPribose) polymerase (PARP), $\mathrm{H}_{2} \mathrm{X}, \mathrm{P}-\mathrm{p} 53, \mathrm{P} 53$, TAKT, T-p38, and $\beta$-actin monoclonal antibody were purchased from Cell Signaling Technology. Terminal deoxynucleotidyl transferase dUTP nick end labeling (TUNEL) assay kit, AnnexinV-FLUOS staining kit, caspase-3 activity assay kit, and BCA protein assay kit were acquired from Beyotime Institute of Biotechnology (Shanghai, People's Republic of China).

\section{Preparation and characterization of Ag@PEI@PTX}

AgNPs were prepared as follows: ${ }^{42}$ briefly, $0.1 \mathrm{~mL}$ of vitamin $\mathrm{C}$ solution $(400 \mu \mathrm{g} / \mathrm{mL})$ was added dropwise into $4 \mathrm{~mL} \mathrm{AgNO}_{3}(400 \mu \mathrm{g} / \mathrm{mL})$ under magnetic stirring for $2 \mathrm{~h}$ at room temperature. Then, AgNPs was mixed in $3.792 \mathrm{mg}$ PEI and $0.32 \mathrm{~mL}$ of $6 \mathrm{mg} / \mathrm{mL}$ PTX at a final volume of 4 mL with Milli-Q water. The Ag@PEI@PTX complex was purified by dialysis for overnight. Ag@PEI@PTX nanoparticles were sonicated and then filtered through a $0.2-\mu \mathrm{m}$ pore size filter. The concentration of AgNPs was measured by inductively coupled plasma atomic emission spectroscopy.AgNPs, Ag@PEI, and Ag@PEI@PTX were characterized by transmission electron microscope (TEM, H-7650; Hitachi). The particle zeta potential and size distribution were determined by Zetasizer Nano ZS (Malvern Instruments Limited) particle analyzer.

\section{Cell culture and viability assay}

The HepG2 and LO2 cells were maintained in Dulbecco's Modified Eagle's Medium supplemented with an antibiotic, fetal bovine serum (10\%), 100 units per $\mathrm{mL}$ penicillin, and 50 units per $\mathrm{mL}$ streptomycin at $37^{\circ} \mathrm{C}$ in a humidified incubator containing 5\% $\mathrm{CO}_{2}$. The cells, proliferative inhibition of $\mathrm{Ag} @$ PEI@PTX nanoparticles was measured using MTT assay as previously described. ${ }^{43}$ Briefly, the cells were incubated with different treatments of control,AgNPs, Ag@PEI, and Ag@ PEI@PTX at a density of $4 \times 10^{4}$ cells per well at $37^{\circ} \mathrm{C}$ for $24 \mathrm{~h}$. Then, $20 \mu \mathrm{L}$ of MTT solution was added to each well $(5 \mathrm{mg} / \mathrm{mL}$ in phosphate-buffered saline [PBS]) and incubated for $5 \mathrm{~h}$. After that, the medium containing MTT was aspirated off and replaced with dimethyl sulfoxide $(150 \mu \mathrm{L} /$ well $)$ to dissolve the dark blue formazan salt formed in surviving cells. The color 
intensity of the formazan solution was read at $570 \mathrm{~nm}$ using a microplate spectrophotometer (SpectroAmax ${ }^{\mathrm{TM}} 250$ ). The cell viability was expressed as percentage of MTT reduction relative to the absorbance of control.

\section{Mitochondrial membrane potential measurement $\left(\Delta \Psi_{m}\right)$}

JC-1 was used to assess the change of mitochondrial membrane potential in HepG2 cells exposed to Ag@PEI@PTX as previously reported. ${ }^{44}$ The cells cultured in 6-well plates were released by trypsinization, resuspended in PBS buffer with $10 \mu \mathrm{g} / \mathrm{mL} \mathrm{JC}-1$, and then incubated at $37^{\circ} \mathrm{C}$ for $30 \mathrm{~min}$. The cells were then harvested by centrifugation, resuspended in PBS, and analyzed by flow cytometry. JC-1 fluorescence was measured using a single excitation wavelength $(485 \mathrm{~nm})$ with dual emission (shift from green at $530 \mathrm{~nm}$ to red at $590 \mathrm{~nm}$ ). The percentage of the green fluorescence from JC-1 monomers was used to represent the cells that lost $\Delta \Psi_{\mathrm{m}}$.

\section{Annexin-V/PI double-staining assay}

Translocation of phosphatidylserine in HepG2 treated with Ag@PEI@PTX was detected by Annexin-V-fluorescein isothiocyanate staining and PI kit as previously described. ${ }^{45}$ In brief, the cells were treated with Ag@PEI@PTX for 24 h and stained with Annexin-V/PI for $30 \mathrm{~min}$, followed by flow cytometric analysis.

\section{Cell-cycle analysis}

The effect of Ag@PEI@PTX on cell-cycle distribution was analyzed by flow cytometry as previously reported. ${ }^{46}$ The cells incubated with Ag@PEI@PTX were collected and centrifuged at a speed of $1,500 \mathrm{rpm}$ for $10 \mathrm{~min}$. Then, the harvested cells were fixed with chilled $70 \%$ ethanol at $-20^{\circ} \mathrm{C}$ overnight. The fixed cells were stained by PI $(50 \mu \mathrm{g} / \mathrm{mL})$ at $37^{\circ} \mathrm{C}$ for $30 \mathrm{~min}$ in darkness. DNA histogram was used to express the proportion of cells in G0/G1, S, and G2/M phases. The apoptotic cells with hypodiploid DNA content were detected by quantifying in the sub-G1 peak.

\section{TUNEL and DAPI staining}

DNA fragmentation induced by Ag@PEI@PTX was detected with fluorescence staining by the TUNEL apoptosis detected kit following the manufacturer's protocol. ${ }^{47}$ Briefly, HepG2 cells in chamber slides were fixed with $3.7 \%$ formaldehyde and then permeabilized with $0.1 \%$ Triton X-100 in PBS. Then, the HepG2 cells were labeled with TUNEL reaction mixture for $1 \mathrm{~h}$ at $37^{\circ} \mathrm{C}$. For nuclear staining, cells were with incubated with $1 \mu \mathrm{g} / \mathrm{mL}$ of DAPI for $30 \mathrm{~min}$ at $37^{\circ} \mathrm{C}$.
Finally, the stained cells were washed with PBS and observed under a fluorescence microscope (Nikon Eclipse 80i).

\section{Caspase-3 activity}

As described in our previous paper, caspase-3 activity was determined by fluorometric method. ${ }^{48}$ Harvested cell pellets were suspended in cell lysis buffer and incubated on ice for $1 \mathrm{~h}$. Caspase activity was determined by fluorescence intensity using caspase-3 substrates (Ac-DEVD-AMC) with an excitation wavelength of $380 \mathrm{~nm}$ and emission wavelength of $460 \mathrm{~nm}$, respectively.

\section{Determination of ROS generation}

ROS accumulation induced by Ag@PEI@PTX-treated HepG 2 cells were estimated by staining cells with DCF fluorescence assay as previously described. ${ }^{42}$ In brief, HepG2 cells were harvested and suspended in PBS containing $10 \mu \mathrm{M}$ of $2^{\prime}, 7^{\prime}$-dichlorofluorescein diacetate for $30 \mathrm{~min}$. ROS generation was indicated by green florescence, which was measured using a microplate reader with an excitation wavelength of $488 \mathrm{~nm}$ and emission wavelength of $525 \mathrm{~nm}$, respectively.

\section{Western blot analysis}

To examine the expression levels of various intracellular proteins in HepG2 cells treated with Ag@PEI@PTX, western blotting was performed as previously reported. ${ }^{49,50}$ Briefly, HepG2 cells treated with Ag@PEI@PTX were incubated with lysis buffer to obtain total cellular proteins, which were then isolated in a $10 \%$ sodium dodecyl sulfate-polyacrylamide gel and transferred onto polyvinylidene fluoride membranes. Later, the polyvinylidene fluoride membranes were incubated with primary antibody and appropriate secondary antibody. The proteins were visualized using ECL chemiluminescence solution and examined on the X-ray film.

\section{Statistical analysis}

Experiments were performed at least for three times. All data were processed using SPSS 19.0 software (IBM Corporation, Armonk, NY, USA). The difference between three or more groups was analyzed by one-way ANOVA multiple comparisons. Differences between two groups were evaluated by two-tailed Student's $t$-test. A probability of $* P<0.05$ or $* * P<0.01$ indicates statistically significant values.

\section{Results and discussion Preparation and characterization of Ag@PEI@PTX}

In this study, a simple method to prepare PEI-modified AgNPs loaded with PTX (Ag@PEI@PTX) to improve anticancer efficacy was demonstrated. The morphology 
and stability of Ag@PEI@PTX was detected and analyzed using various methods. TEM images showed that Ag@ PEI@PTX presented a spherical and monodisperse particles (Figure 1A). The zeta potential of AgNPs was $-17.8 \mathrm{mV}$ and increased to $23 \mathrm{mV}$ after capping with PEI and PTX (Figure 1B), which indicated that Ag@PEI@PTX with positive charge was easier to cross into the cell membrane. Ag@PEI@PTX presented high uniformity with a minimum diameter of $2 \mathrm{~nm}$ (Figure 1C). Furthermore, the size distribution of Ag@PEI@PTX revealed that the decorated AgNPs was stable at least 30 days (Figure 1D), which also indicated that Ag@PEI@PTX was highly stable in aqueous solutions. Taken together, with the property of smart, stable, and zeta potential positive charge, Ag@PEI@PTX could easily enter into cells.

\section{In vitro cytoxicity of Ag@PEI@PTX}

To determine the cell viability and reflect their growth states, the anticancer activity of AgNPs,Ag@PEI, and Ag@PEI@ PTX was measured by MTT assay. As shown in Figure 2A, the cell viability of HepG2 was dramatically lower than LO2 cells when treated with AgNPs (81.27\% vs 96.67\%), Ag@PEI (71.86\% vs 88.45\%), and Ag@PEI@PTX (77.21\% vs 58.32\%). Compared with AgNPs and Ag@PEI, Ag@ PEI@PTX significantly inhibited the growth of HepG2 cells and exhibited low cytotoxic toward LO2 cells. As shown in Figure 2B, the effects of AgNPs,Ag@PEI, and Ag@PEI@ PTX on the growth of HepG2 cells were further confirmed. After being treated with AgNPs,Ag@PEI, and Ag@PEI@ PTX, the cell numbers reduced with cells rounding and cytoplasm shrinkage. The results suggested that Ag@PEI@ PTX effectively inhibited the proliferation of HepG2 cells and induced cancer cell death.

\section{Depletion of mitochondrial membrane potential $\left(\Delta \Psi_{m}\right)$}

Mitochondrial membrane potential $\left(\Delta \Psi_{\mathrm{m}}\right)$ by flow cytometric analysis was used to investigate the initiation of apoptosis. ${ }^{44}$ As shown in Figure 3A, compared with the control group, the mitochondrial membrane potential was reduced significantly to $87.1 \%, 53.4 \%$, and $36.5 \%$ for AgNPs, Ag@PEI, and Ag@PEI@PTX, respectively. As shown in Figure 3B, representative scattergrams for red-FL2-H fluorescence indicated an intact mitochondrial membrane potential and green-FL1-H fluorescence indicated the loss of membrane potential. Treatment of HepG2 cells resulted in elevation of mitochondria depolarization, which is indicated by the shift of fluorescence from red to green. These results demonstrated that Ag@PEI@PTX triggered HepG2 cells apoptosis through the induction of mitochondrial dysfunction.
A
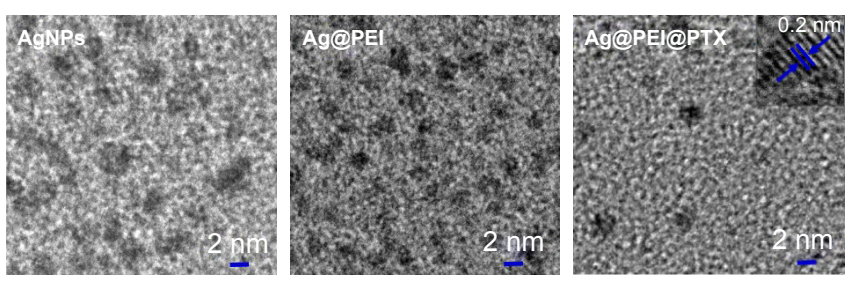

B

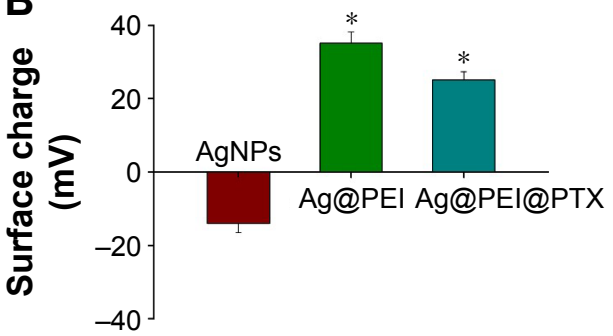

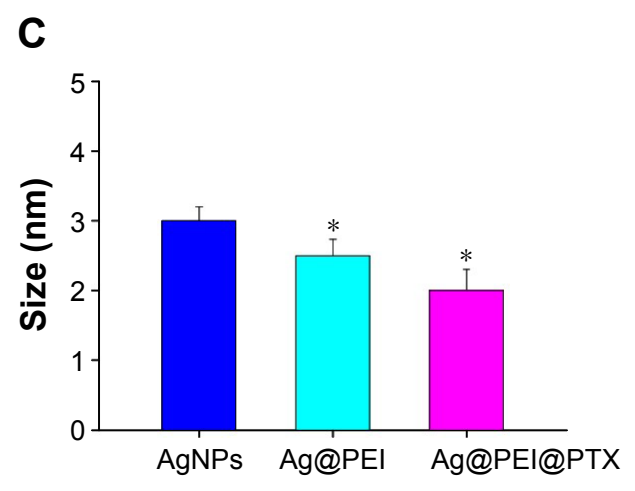

D

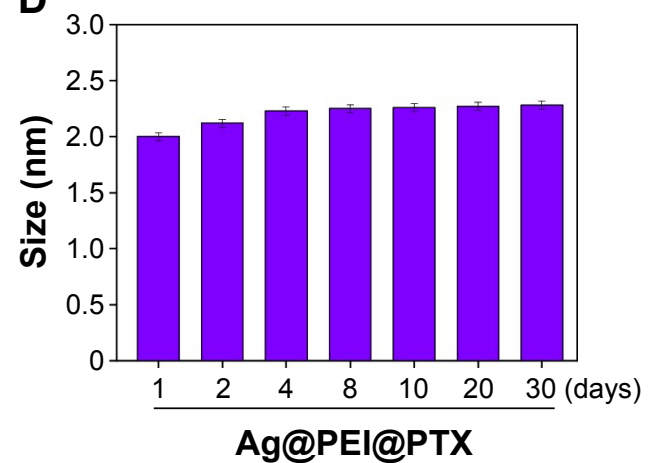

Figure I Characterization of Ag@PEI@PTX.

Notes: (A) Transmission electron microscopy imaging of AgNPs, Ag@PEl, and Ag@PEI@PTX. Inset is a TEM image of an individual particle, lattice fringe. (B) Zeta potential of AgNPs, Ag@PEl, and Ag@PEI@PTX. (C) Particle size of AgNPs, Ag@PEl, and Ag@PEI@PTX. (D) Stability of Ag@PEI@PTX in aqueous solutions. Bars with different characters are statistically different at $P<0.05$ (*) level.

Abbreviations: AgNPs, silver nanoparticles; PEI, polyethylenimine; PTX, paclitaxel. 

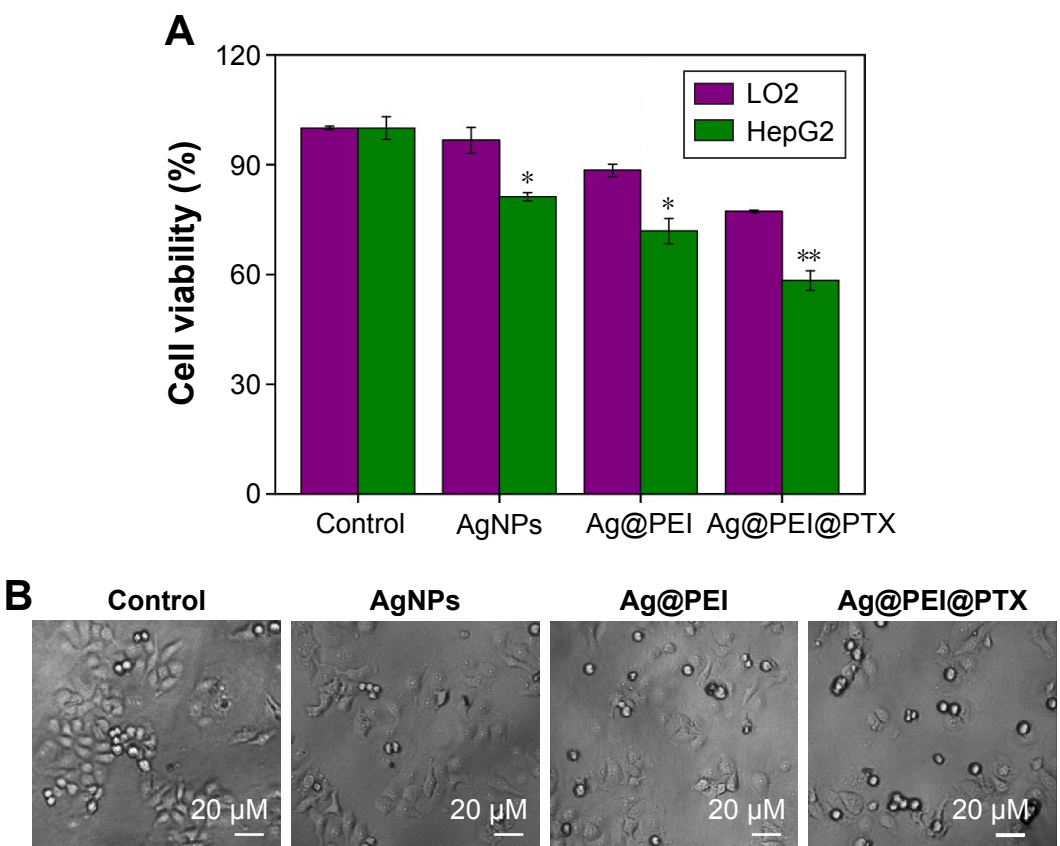

Figure 2 Effects of AgNPs, Ag@PEl, and Ag@PEI@PTX on the growth of HepG2.

Notes: (A) Cell viability was evaluated after treated with AgNPs, Ag@PEl, and Ag@PEI@PTX for 24 h by MTT assay. The concentration of AgNPs, Ag@PEl, and Ag@PEI@PTX was $2.5 \mu \mathrm{g} / \mathrm{mL}$. (B) Morphological changes in HepG2 cells with different treatments. Bars with different characters are statistically different at $P<0.05$ (*) or $P<0.01$ (**) level. Abbreviations: NPs, nanoparticles; MTT, 3-(4,5-dimethylthiazol-2-yl)-2,5-diphenyltetrazolium bromide; PEl, polyethylenimine; PTX, paclitaxel.
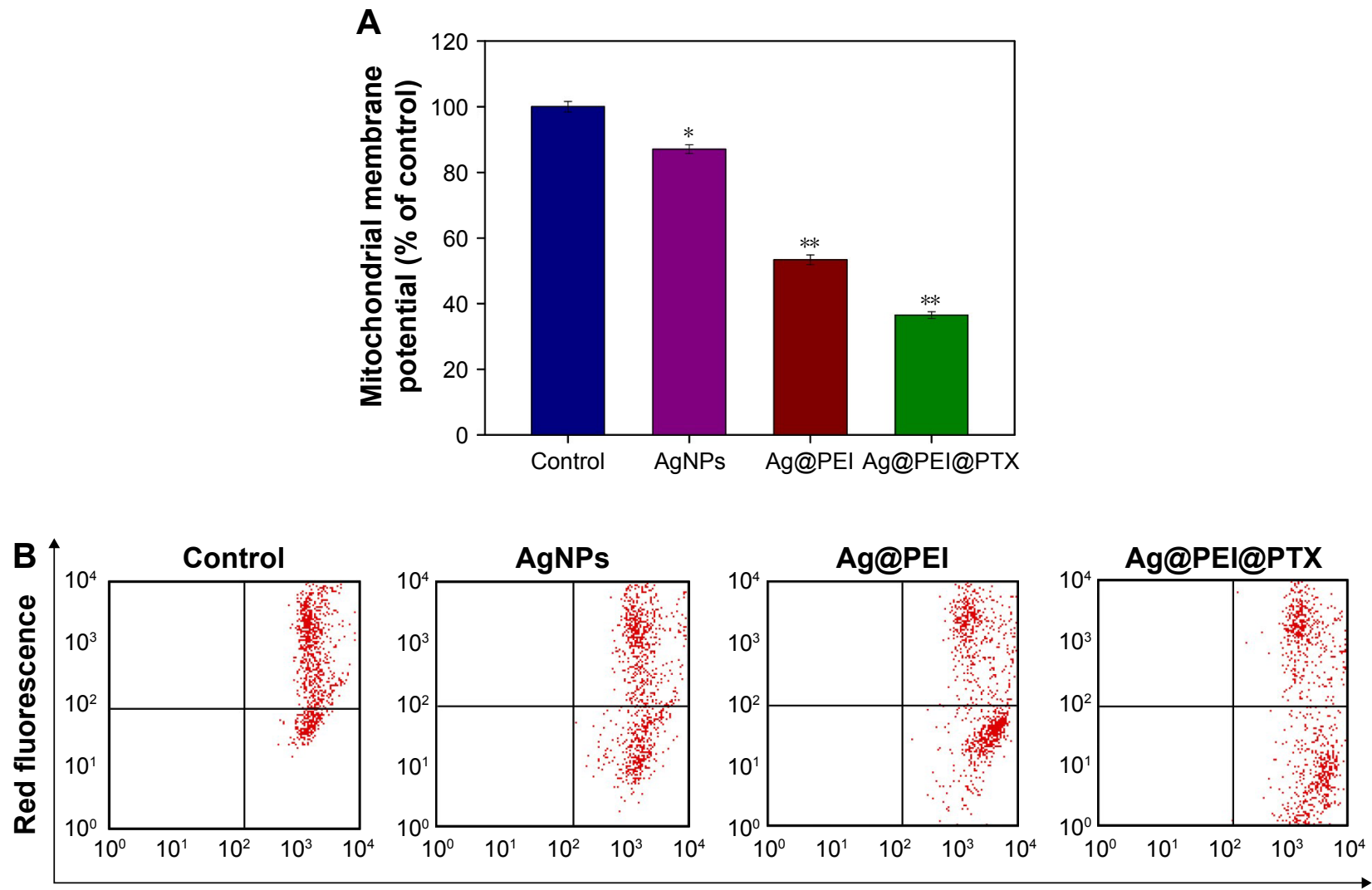

\section{Green fluorescence}

Figure 3 Depletion of mitochondrial membrane potential induced by AgNPs, Ag@PEl, and Ag@PEl@PTX.

Notes: (A) The percentage of mitochondrial membrane potential. (B) Mitochondrial membrane potential of HepG2 exposed to AgNPs, Ag@PEl, and Ag@PEl@PTX. Bars with different characters are statistically different at $P<0.05$ (*) or $P<0.01$ (**) level.

Abbreviations: AgNPs, silver nanoparticles; PEI, polyethylenimine; PTX, paclitaxel. 


\section{Translocation of phosphatidylserine induced by Ag@PEI@PTX}

The translocation of phosphatidylserine to the outer membrane is an important step in the apoptosis of HepG2 cells. ${ }^{44}$ Annexin-V/PI double-staining assay was also used to characterize the apoptotic cells death induced by $\mathrm{Ag} @$ PEI@PTX. As shown in Figure 4, dot plot results of HepG2 cell treated groups showed the presence of both early and late apoptotic cells. HepG2 cells treated with Ag@PEI@ PTX revealed the increased cell number of apoptosis. Taken together, the results indicated that Ag@PEI@PTX inhibited HepG2 cells proliferation mainly through apoptosis.

\section{Ag@PEI@PTX induced HepG2 cell apoptosis}

Apoptosis was one of the most crucial mechanisms accounting for the anticancer action. ${ }^{44}$ In this study, PI-flow cytometric analysis was used to investigate whether apoptosis was involved in the cell death induced by AgNPs, Ag@PEI, and Ag@PEI@PTX. As shown in Figure 5A, compared with different treatments, the sub-G1 apoptotic cell population of Ag@PEI@PTX was significantly increased in the DNA histogram. For instance, the exposure of HepG2 cells to different treatments of control,AgNPs, Ag@PEI, and Ag@ PEI@PTX significantly increased in apoptotic population from $1.05 \%$ to $17.62 \%$, while no significant change in $\mathrm{G} 0 / \mathrm{G} 1, \mathrm{~S}$, and $\mathrm{G} 2 / \mathrm{M}$ phases was observed. DNA fragmentation is an important biochemical hallmark of cell apoptosis. The induction of apoptosis was further confirmed by TUNEL enzymatic labeling and DAPI co-staining assay. As shown in Figure 5B, HepG2 cells exhibited typical apoptotic features with Ag@PEI@PTX, such as DNA fragmentation and nuclear condensation. These results demonstrated that $\mathrm{Ag} @$ PEI@PTX induced HepG2 cell apoptosis.

\section{Induction of caspase-mediated PARP cleavage by Ag@PEI@PTX}

Caspase- 3 has been considered as the primary executioner of apoptosis, as it is responsible for the cleavage of many proteins and caspase involved in programmed cells death. PARP, which is one of the main cleavage targets of caspase-3, is downstream of caspase family proteins in apoptosis pathways.

Caspase-3 and the subsequent cleavage of PARP were examined by western blotting to evaluate their involvement and contribution to cell apoptosis. As shown in Figure 6A, compared with the control group, AgNPs, and $\mathrm{Ag} @ \mathrm{PEI}$, treatments of HepG2 cells with Ag@PEI@PTX significantly increased the activity of caspase-3. As shown in Figure 6B, the expression level of caspase-3 and PARP was downregulated with different treatments. The results show that Ag@PEI@PTX significantly strengthened the activation of caspase- 3 and the cleavage of downstream effect PARP. Taken together, these results reveal that the nanosystem significantly inhibits cancer cell growth by inducing apoptosis.

\section{Induction of ROS generation by Ag@PEI@PTX}

ROS was considered as an important regulator of cell apoptosis, especially that induced by anticancer drugs. Variety of factors can cause damage to the structure and function of mitochondria and further induce cell apoptosis. Mitochondrium is a major place to produce intracellular ROS. ${ }^{51}$ To investigate whether Ag@PEI@PTX could trigger ROS-mediated apoptosis, the intracellular ROS level was determined by DCF fluorescence assay. As shown in Figure 7A, compared with control group, AgNPs, and $\mathrm{Ag} @$ PEI, the ROS generation of HepG2 cells increased
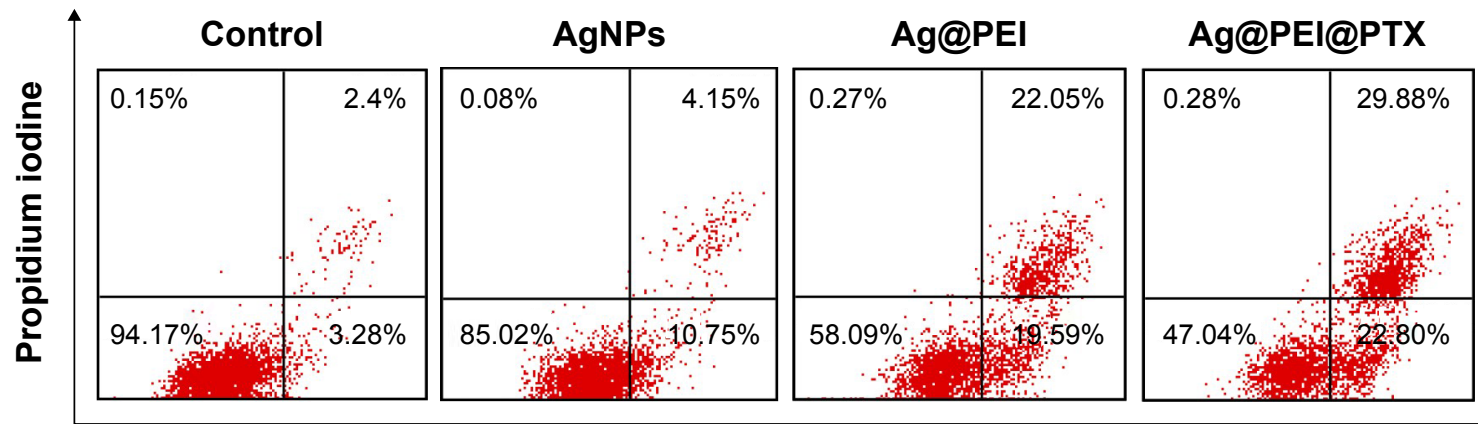

Annexin V-FITC

Figure 4 Translocation of phosphatidylserine induced by AgNPs, Ag@PEl, and Ag@PEI@PTX in HepG2 cells.

Note: The upper right quadrant indicated cells in the early stage of apoptosis and lower right shows cells in the late stage of apoptosis or necrosis.

Abbreviations: AgNPs, silver nanoparticles; FITC, fluorescein isothiocyanate; PEI, polyethylenimine; PTX, paclitaxel. 

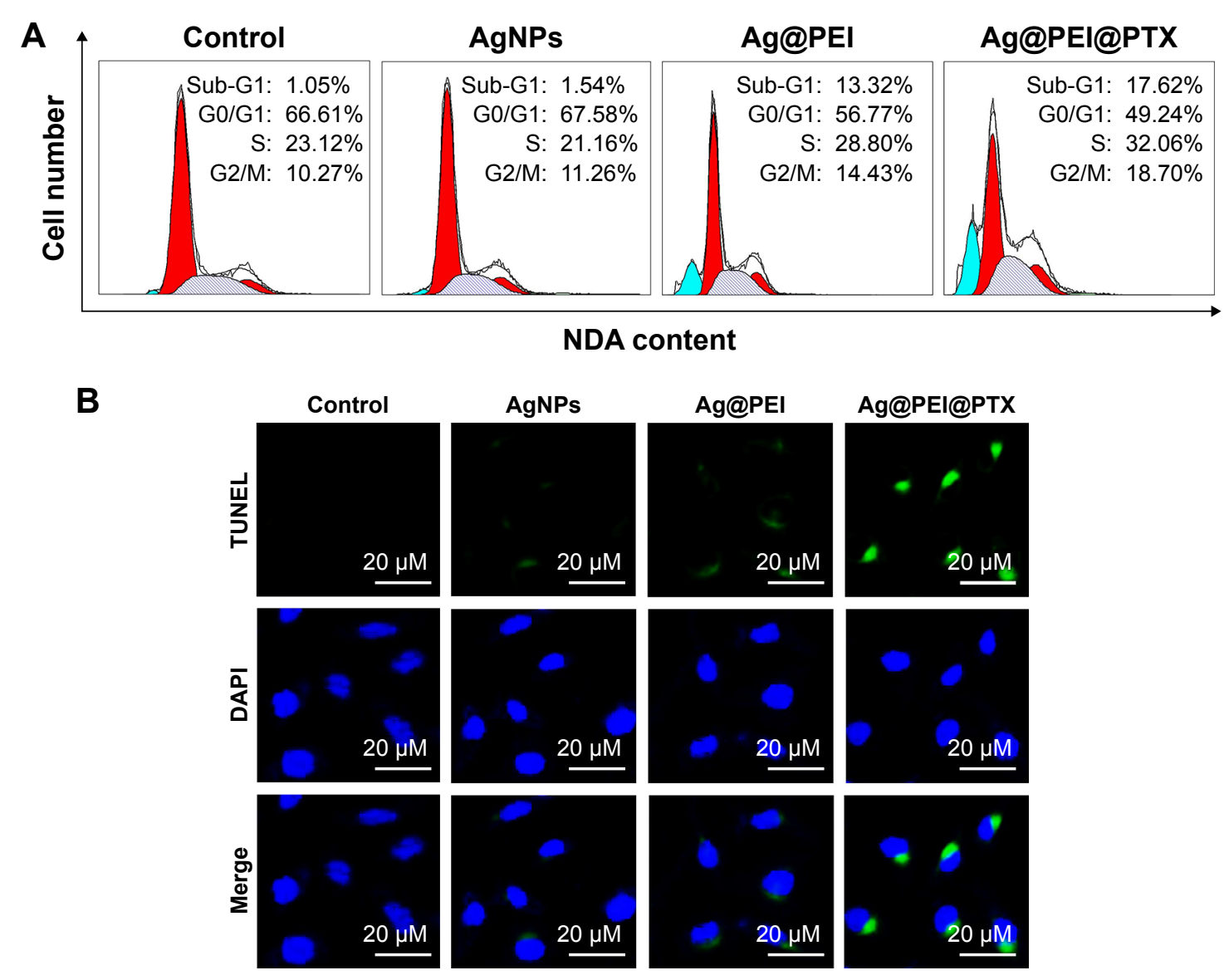

Figure 5 Ag@PEI@PTX-induced apoptosis in HepG2 cells.

Notes: (A) The cell-cycle distribution with different treatments was analyzed by quantifying DNA content using flow cytometric analysis. (B) Representative photomicrographs of DNA fragmentation and nuclear condensation as detected by TUNEL-DAPI co-staining assay.

Abbreviations: AgNPs, silver nanoparticles; DAPI, 4'6-diamidino-2-phenylindole; TUNEL, terminal transferase dUTP nick end labeling.

significantly after treatment with Ag@PEI@PTX. As shown in Figure 7B, the fluorescent intensity of DCF in HepG2 cells exposure to Ag@PEI@PTX was the most strongest in treatment groups. These results indicate the involvement of ROS in the anticancer activity of Ag@PEI@PTX.

\section{Activation of ROS-mediated signaling pathways byAg@PEI@PTX}

The mechanism of apoptosis by Ag@PEI@PTX induced in HepG2 cells was further investigated. Intracellular ROS overproduction could trigger DNA damage and cause a series of different signaling pathways, such as p53, AKT, and MAPK signaling pathways. Due to the detection of ROS overproduction in cells exposed to Ag@PEI@PTX, western blotting was used to examine the effects on the ROS-mediated signaling pathway. As shown in Figure 8A, compared with AgNPs and Ag@PEI, the expression of total AKT was downregulated after treatment with Ag@PEI@ PTX. Meanwhile, as shown in Figure 8B, HepG2 cells treated
withAg@PEI@PTX effectively increased the expression of total proapoptotic kinase p38 in HepG2 cells. For the p53 signaling pathway, Ag@PEI@PTX significantly increased the expression levels of $\mathrm{H}_{2} \mathrm{X}, \mathrm{p}-\mathrm{p} 53$, and total p53 (Figure 8C). Taken together, these results reveal that nanosystem induces HepG2 cells apoptosis through regulation of ROS-mediated AKT, MAPK, and p53 signaling pathways (Figure 8D).

\section{Conclusion}

In summary, the present study demonstrated that PEI-modified AgNPs loaded with PTX were successfully fabricated. Ag@ PEI@PTX showed a dramatic cytotoxic effects of HepG2 cells, which enhanced the drug sensitivity and induced apoptosis of cancer cells rather than normal cells. Moreover, the underlying molecular mechanisms indicated that Ag@PEI@PTX activated caspase-3-mediated HepG2 cell apoptosis via ROS generation. Furthermore, our results revealed the apoptotic signaling pathway through ROS was triggered by the Ag@PEI@PTX in HepG2 cells, including 

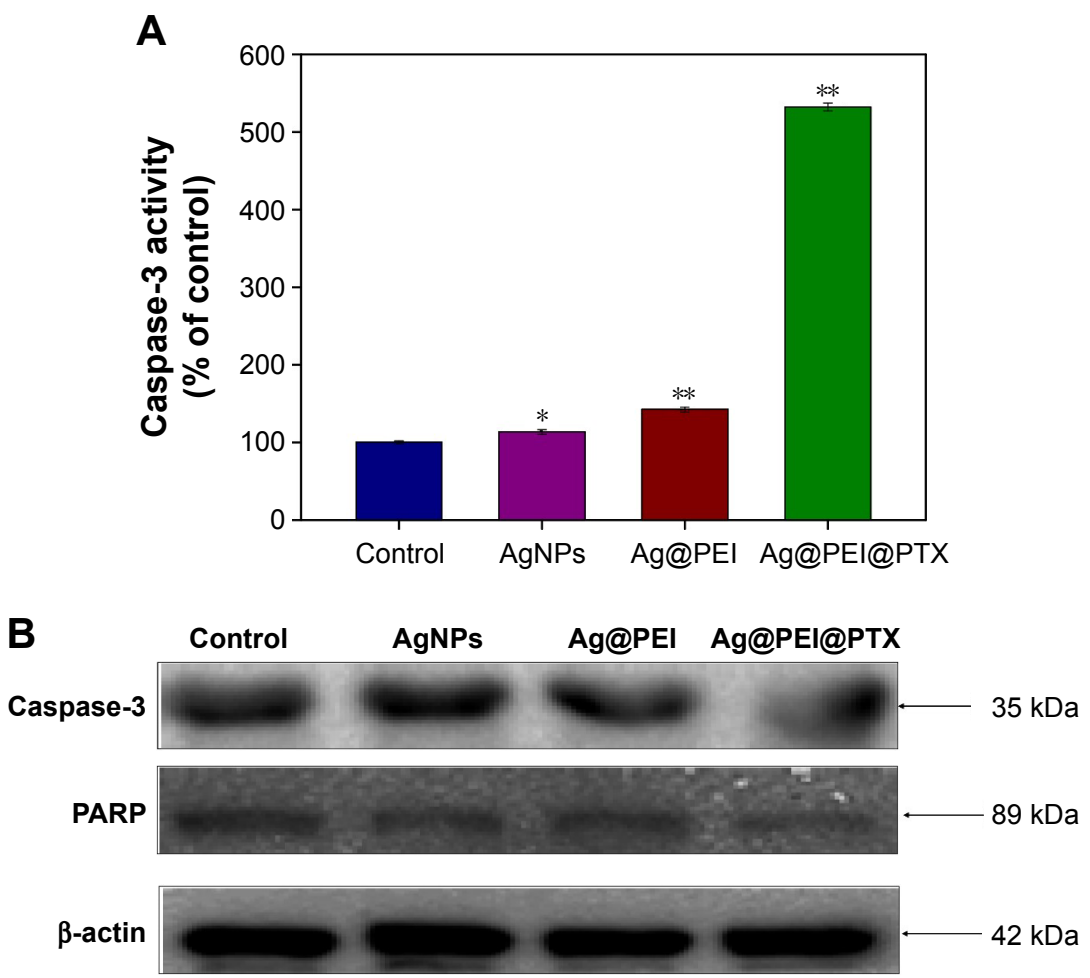

Figure 6 Caspase-3 and PARP-mediated apoptosis induced by Ag@PEI@PTX in HepG2 cells.

Notes: (A) HepG2 cells treated with Ag@PEI@PTX and caspase-3 activity were analyzed by synthetic fluorogenic substrate. (B) The expression of PARP and caspase-3 by Western blot; $\beta$-actin was used as the loading control. Bars with different characters are statistically different at $P<0.05(*)$ or $P<0.01 \quad(* *)$ level.

Abbreviations: AgNPs, silver nanoparticles; PARP, poly(ADP-ribose) polymerase.

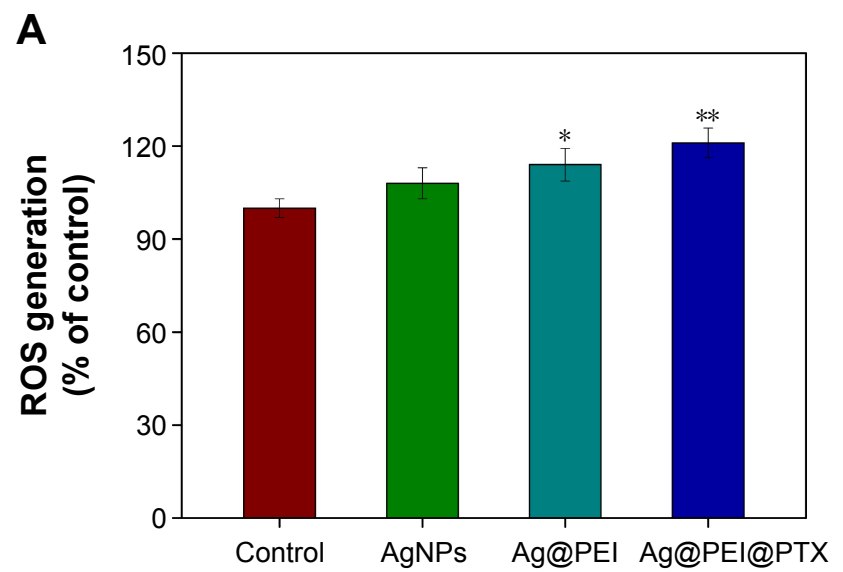

B Control

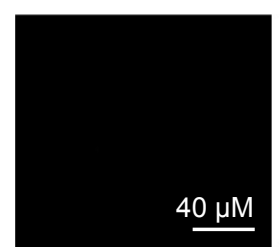

AgNPs

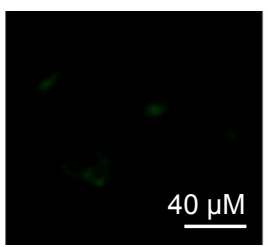

Ag@PEI

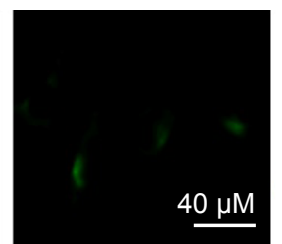

Ag@PEI@PTX

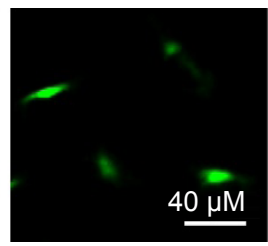

Figure 7 ROS overproduction induced by Ag@PEI@PTX of HepG2 cells.

Notes: (A) Changes in intracellular ROS generation detected by measuring DCF fluorescence intensity. (B) HepG2 cells were incubated with I0 $\mu$ M DCF-DA in PBS for 30 min and then treated with AgNPs, Ag@PEI, and Ag@PEI@PTX under microscope. Bars with different characters are statistically different at $* P<0.05$ or $* * P<0.01$. Abbreviations: AgNPs, silver nanoparticles; DCF-DA, 2',7'-dichlorofluorescein diacetate; PBS, phosphate buffered saline; ROS, reactive oxygen species. 
A

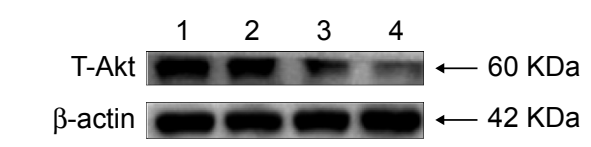

B

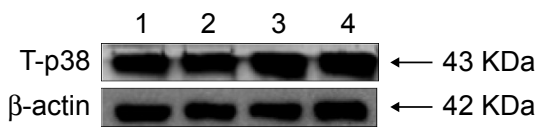

C

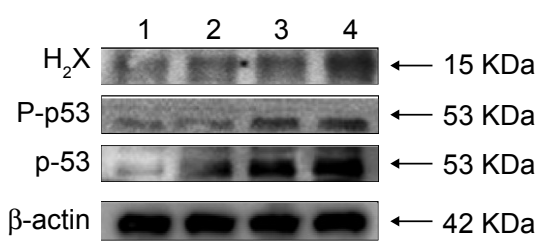

D

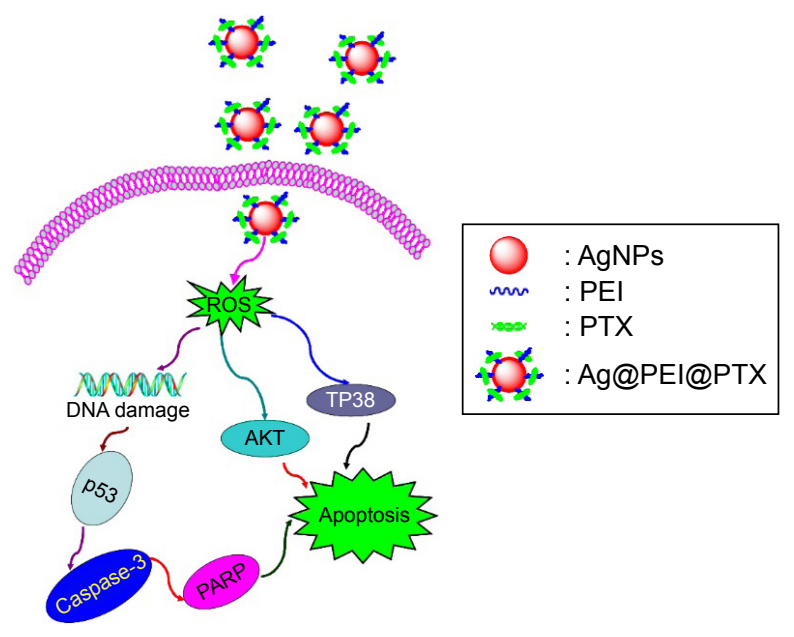

(1: control 2: AgNPs 3: Ag@PEI 4: Ag@PEI@PTX)

Figure 8 Activation of intracellular apoptotic signaling pathways by Ag@PEI@PTX in HepG2 cells.

Notes: (A) Phosphorylation status expression levels of AKT pathways. (B) Phosphorylation status expression levels of T-p38 MAPK pathways. (C) Activation of p53 signaling pathway. (D) The signaling pathway of regulation of ROS-mediated p53, AKT, and MAPK signaling pathways. The expression of $\beta$-actin was measured as control.

Abbreviations: AgNPs, silver nanoparticles; PARP, poly(ADP-ribose) polymerase; PEl, polyethyleneimine; PTX, paclitaxel; ROS, reactive oxygen species.

AKT, MAPK, and p53 signaling pathways. Taken together, this study provides the possibility of using nanosystem to induce HepG2 cell apoptosis and offers a new strategy for potential cancer therapy.

\section{Acknowledgments}

This work was supported by the China Postdoctoral Science Foundation (2015M582366), the Technology Planning Project of Guangdong Province (No: 2014A020212697), the Technology Planning Project of Guangzhou (No: 201607010120), and the Guangzhou Medical Health Science and Technology Project (No: 20151A010051).

\section{Disclosure}

The authors report no conflicts of interest in this work.

\section{References}

1. Scortegagna E Jr, Karam AR, Sioshansi S, Bozorgzadeh A, Barry C, Hussain S. Hepatocellular carcinoma recurrence pattern following liver transplantation and a suggested surveillance algorithm. Clin Imaging. 2016;40(6):1131-1134.

2. Dong Y, Zou JJ, Su S, et al. MicroRNA-218 and microRNA-520a inhibit cell proliferation by downregulating E2F2 in hepatocellular carcinoma. Mol Med Rep. 2015;12(1):1016-1022.

3. Torre LA, Bray F, Siegel RL, Ferlay J, Lortet-Tieulent J, Jemal A. Global cancer statistics, 2012. CA Cancer J Clin. 2015;65(2):87-108.

4. Gong XL, Qin SK. Progress in systemic therapy of advanced hepatocellular carcinoma. World J Gastroentero. 2016;22(29):6582-6594.

5. Xia HP, Chen JX, Shi M, Deivasigamani A, Ooi LL, Hui KM. The overexpression of survivin enhances the chemotherapeutic efficacy of YM155 in human hepatocellular carcinoma. Oncotarget. 2015;6(8):5990-6000.
6. Ling D, Xia H, Park W, et al. pH-sensitive nanoformulated triptolide as a targeted therapeutic strategy for hepatocellular carcinoma. ACS Nano. 2014;8(8):8027-8039.

7. Thapa RK, Choi JY, Poudel BK, et al. Multilayer-coated liquid crystalline nanoparticles for effective sorafenib delivery to hepatocellular carcinoma. ACS Appl Mater Interfaces. 2015;7(36):20360-20368.

8. Rand D, Ortiz V, Liu YA, et al. Nanomaterials for X-ray imaging: gold nanoparticle enhancement of X-ray scatter imaging of hepatocellular carcinoma. Nano Lett. 2011;11(7):2678-2683.

9. Li JH, Sharkey CC, Huang DT, King MR. Nanobiotechnology for the therapeutic targeting of cancer cells in blood. Cell Mol Bioeng. 2015; $8(1): 137-150$.

10. Friberg S, Nystrom AM. NANOMEDICINE: Will it offer possibilities to overcome multiple drug resistance in cancer? J Nanobiotechnol. 2016; 14:1-17.

11. Yuan B, Webster TJ, Roy AK. Cytoprotective effects of cerium and selenium nanoparticles on heat-shocked human dermal fibroblasts: an in vitro evaluation. Int J Nanomed. 2016;11:1427-1433.

12. Chauhan VP, Jain RK. Strategies for advancing cancer nanomedicine. Nat Mater. 2013;12(11):958-962.

13. Abbai R, Mathiyalagan R, Markus J, et al. Green synthesis of multifunctional silver and gold nanoparticles from the oriental herbal adaptogen: Siberian ginseng. Int J Nanomed. 2016;11:3131-3143.

14. Hassan CE, Webster TJ. The effect of red-allotrope selenium nanoparticles on head and neck squamous cell viability and growth. Int $J$ Nanomed. 2016;11:3641-3654.

15. Wang YZ, Fan Z, Shao L, et al. Nanobody-derived nanobiotechnology tool kits for diverse biomedical and biotechnology applications. Int $J$ Nanomed. 2016;11:3287-3302.

16. Yang XX, Li CM, Huang CZ. Curcumin modified silver nanoparticles for highly efficient inhibition of respiratory syncytial virus infection. Nanoscale. 2016;8(5):3040-3048.

17. He Y, Du Z, Ma S, et al. Biosynthesis, antibacterial activity and anticancer effects against prostate cancer (PC-3) cells of silver nanoparticles using Dimocarpus longan lour. peel extract. Nanoscale Res lett. 2016; 11(1):300.

18. Jeong JK, Gurunathan S, Kang MH, et al. Hypoxia-mediated autophagic flux inhibits silver nanoparticle-triggered apoptosis in human lung cancer cells. Sci Rep. 2016;6:1-13. 
19. Dziedzic A, Kubina R, Buldak RJ, Skonieczna M, Cholewa K. Silver nanoparticles exhibit the dose-dependent anti-proliferative effect against human squamous carcinoma cells attenuated in the presence of berberine. Molecules. 2016;21(3):365.

20. Li YH, Lin ZF, Zhao MQ, et al. Multifunctional selenium nanoparticles as carriers of HSP70 siRNA to induce apoptosis of HepG2 cells. Int J Nanomed. 2016;11:3065-3076.

21. Liu YJ, Zhang B, Yan B. Enabling anticancer therapeutics by nanoparticle carriers: the delivery of paclitaxel. Int J Mol Sci. 2011;12(7): 4395-4413.

22. Ye J, Xia X, Dong W, et al. Cellular uptake mechanism and comparative evaluation of antineoplastic effects of paclitaxel-cholesterol lipid emulsion on triple-negative and non-triple-negative breast cancer cell lines. Int J Nanomed. 2016;11:4125-4140.

23. Voloshin T, Munster M, Blatt R, et al. Alternating electric fields (TTFields) in combination with paclitaxel are therapeutically effective against ovarian cancer cells in vitro and in vivo. Int Cancer. 2016;139(12): 2850-2858.

24. Yue $\mathrm{T}$, Zheng $\mathrm{X}$, Dou $\mathrm{Y}$, et al. Interleukin 12 shows a better curative effect on lung cancer than paclitaxel and cisplatin doublet chemotherapy. BMC Cancer. 2016;16:665.

25. Barker HE, Patel R, McLaughlin M, et al. CHK1 inhibition radiosensitizes head and neck cancers to paclitaxel-based chemoradiotherapy. Mol Cancer Ther. 2016;15(9):2042-2054.

26. Simon-Gracia L, Hunt H, Scodeller $P$, et al. iRGD peptide conjugation potentiates intraperitoneal tumor delivery of paclitaxel with polymersomes. Biomaterials. 2016;104:247-257.

27. Tanei T, Leonard F, Liu XW, et al. Redirecting transport of nanoparticle albumin-bound paclitaxel to macrophages enhances therapeutic efficacy against liver metastases. Cancer Res. 2016;76(2):429-439.

28. Horinouchi H, Yamamoto N, Nokihara H, et al. A phase 1 study of linifanib in combination with carboplatin/paclitaxel as first-line treatment of Japanese patients with advanced or metastatic non-small cell lung cancer (NSCLC). Cancer Chemoth Pharm. 2014;74(1):37-43.

29. Xu JH, Hu SL, Shen GD, Shen G. Tumor suppressor genes and their underlying interactions in paclitaxel resistance in cancer therapy. Cancer Cell Int. 2016;16:13.

30. Parayath NN, Nehoff H, Norton SE, et al. Styrene maleic acidencapsulated paclitaxel micelles: antitumor activity and toxicity studies following oral administration in a murine orthotopic colon cancer model. Int J Nanomed. 2016;11:3979-3991.

31. He ZJ, Wan XM, Schulz A, et al. A high capacity polymeric micelle of paclitaxel: implication of high dose drug therapy to safety and in vivo anti-cancer activity. Biomaterials. 2016;101:296-309.

32. Choi EO, Park C, Hwang HJ, et al. Baicalein induces apoptosis via ROSdependent activation of caspases in human bladder cancer 5637 cells. Int J Oncol. 2016;49(3):1009-1018.

33. Liu K, Zhao Q, Liu P, et al. ATG3-dependent autophagy mediates mitochondrial homeostasis in pluripotency acquirement and maintenance. Autophagy. 2016;12(11):1-9.

34. Zhang XF, Gurunathan S. Combination of salinomycin and silver nanoparticles enhances apoptosis and autophagy in human ovarian cancer cells: an effective anticancer therapy. Int J Nanomed. 2016;11:3655-3675.

35. Khojah HM, Ahmed S, Abdel-Rahman MS, Hamza AB. Reactive oxygen and nitrogen species in patients with rheumatoid arthritis as potential biomarkers for disease activity and the role of antioxidants. Free Radic Bio Med. 2016;97:285-291.

International Journal of Nanomedicine

\section{Publish your work in this journal}

The International Journal of Nanomedicine is an international, peerreviewed journal focusing on the application of nanotechnology in diagnostics, therapeutics, and drug delivery systems throughout the biomedical field. This journal is indexed on PubMed Central, MedLine, CAS, SciSearch $®$, Current Contents ${ }^{\circledR} /$ Clinical Medicine,
36. Farah MA, Ali MA, Chen SM, et al. Silver nanoparticles synthesized from Adenium obesum leaf extract induced DNA damage, apoptosis and autophagy via generation of reactive oxygen species. Colloid Surface B Biointerfaces. 2016;141:158-169.

37. Di Meo S, Reed TT, Venditti P, Victor VM. Role of ROS and RNS sources in physiological and pathological conditions. Oxid Med Cell Longev. 2016;2016:1245049.

38. Zhang JX, Wang XL, Vikash V, et al. ROS and ROS-mediated cellular signaling. Oxid Med Cell Longev. 2016;2016:4350965.

39. Giacco F, Du XL, Carratu A, et al. GLP-1 cleavage product reverses persistent ROS generation after transient hyperglycemia by disrupting an ROS-generating feedback loop. Diabetes. 2015;64(9):3273-3284.

40. Lee MJ, Lee SJ, Yun SJ, et al. Silver nanoparticles affect glucose metabolism in hepatoma cells through production of reactive oxygen species. Int J Nanomed. 2016;11:55-68.

41. Sun C, Yin NY, Wen RX, et al. Silver nanoparticles induced neurotoxicity through oxidative stress in rat cerebral astrocytes is distinct from the effects of silver ions. Neurotoxicology. 2016;52:210-221.

42. Zhu B, Li Y, Lin Z, et al. Silver nanoparticles induce HePG-2 cells apoptosis through ROS-mediated signaling pathways. Nanoscale Res Lett. 2016;11(1):198.

43. You YY, Hu H, He LZ, Chen T. Differential effects of polymer-surface decoration on drug delivery, cellular retention, and action mechanisms of functionalized mesoporous silica nanoparticles. Chem Asian J. 2015; 10(12):2743-2753.

44. Zhang YB, Li XL, Huang Z, Zheng W, Fan C, Chen T. Enhancement of cell permeabilization apoptosis-inducing activity of selenium nanoparticles by ATP surface decoration. Nanomedicine. 2013;9(1):74-84.

45. Wu HL, Li XL, Liu W, et al. Surface decoration of selenium nanoparticles by mushroom polysaccharides-protein complexes to achieve enhanced cellular uptake and antiproliferative activity. J Mater Chem. 2012;22(19):9602-9610.

46. Li YH, Li XL, Zheng WJ, Fan C, Chen T. Functionalized selenium nanoparticles with nephroprotective activity, the important roles of ROS-mediated signaling pathways. J Mater Chem B. 2013;1(46):6365-6372.

47. Li YH, Li XL, Wong YS, et al. The reversal of cisplatin-induced nephrotoxicity by selenium nanoparticles functionalized with 11-mercapto1-undecanol by inhibition of ROS-mediated apoptosis. Biomaterials. 2011;32(34):9068-9076.

48. Liu W, Li XL, Wong YS, et al. Selenium nanoparticles as a carrier of 5-Fluorouracil to achieve anticancer synergism. ACS Nano. 2012;6(8): 6578-6591.

49. Huang YY, He LZ, Liu W, et al. Selective cellular uptake and induction of apoptosis of cancer-targeted selenium nanoparticles. Biomaterials. 2013;34(29):7106-7116.

50. Li Y, Lin Z, Zhao M, et al. Silver nanoparticle based codelivery of oseltamivir to inhibit the activity of the H1N1 influenza virus through ROS-mediated signaling pathways. ACS Appl Mater Interfaces. 2016; 8(37):24385-24393.

51. Chen XF, Song MJ, Zhang B, Zhang Y. Reactive oxygen species regulate $\mathrm{T}$ cell immune response in the tumor microenvironment. Oxid Med Cell longev. 2016;2016:1580967.

Journal Citation Reports/Science Edition, EMBase, Scopus and the Elsevier Bibliographic databases. The manuscript management system is completely online and includes a very quick and fair peer-review system, which is all easy to use. Visit http://www.dovepress.com/ testimonials.php to read real quotes from published authors. 